\title{
Design and Implementation of Intelligent Fire Notification Service Using IP Camera in Smart Home
}

\author{
Lei Hang ${ }^{1}$ and Do-Hyeun $\mathrm{Kim}^{2}$ \\ ${ }^{1}$ Computer Engineering Department, \\ Jeju National University, South Korea \\ 1hanglei112233@hotmail.com, ${ }^{2}$ kimdh@jejunu.ac.kr
}

\begin{abstract}
The fire detection is a strong interest for the home surveillance services the smart home. We have a great potential to reduce the devastation caused the fire, and use the information through image processing and information technology in fire detection. This paper presents the intelligent fire push notification service based on IP camera and Web application server with real time processing practically for the mobile user in smart home. We support an automatic fire identification based on the camera image and to alert the fire situation, and to warn the mobile user consequently. And, we design and implement the fire detection using flame color feature extraction of the IP camera images and a push notification mechanism based on IETF HTTP protocol for the mobile user. Also, we evaluate the processing time of the fire detection and push notification to verify the performance of the proposed service. The proposed fire push notification service is intended to improve the home surveillance in terms of practical mobile approach for the safety home environments.
\end{abstract}

Keywords: Fire push notification, Color feature extraction, IP camera, Smart home, Video surveillance.

\section{Introduction}

With the rapid development of information technology, the video surveillance system or the home monitoring system has been widely adopted in the smart home. The video camera can be the key device of this arrangement, obviously, through which it can be used for different purposes, but above all, to obtain an intruder in the house and have the registry of the surroundings for its later visualization. Among all the purposes in the smart house, fire is considered as one of the most important and essential resources due to the enormous perniciousness for the occupants and the infrastructure brought by the fire [1]. The conventional fire detection system using smoke and temperature sensors has many shortcomings such as high false rates and there is a strong demand to design a new approach for the fires detection with lower false rates. Visual information, such as activity monitoring and object tracking, intrusion detection system and video surveillance systems aims to recognize fire occurrence events in any situations. Furthermore, it is important to give notice to the owners of the corresponding department about the actual conditions of the fire outbreak shortly. Many CCTV camera based fire detection applications that exploit a surveillance network has been diffusely used in public places, such as the smart home network and the prevention of forest fires, where conventional fire detection sensors cannot be used [2]. To the best of my knowledge, most of these systems utilize alarms to warn users when a fire occurs. However, these systems have an obvious disadvantage that if the users are far away from the alarms, he may be unable to identify the fire occurs. Some systems utilize GSM to send fire notification but GSM mobile is not always reliable

Received (October 15, 2017), Review Result (December 19, 2017), Accepted (January 16, 2018) 
due to network problems and the power requirement issues. With the development of mobile technology, more and more people are starting to get online on their phones.

In this paper, we propose an intelligent mobile fire notification service in smart home, which is a Web server application and uses the Internet for communication. We design and implement a push notification service to deliver a security alert to the relevant mobile owners. The Web server communicates to the related mobile devices and at the same time users can monitor the videos that contain the fire behavior in their smart phones. The utilized fire detection approach and push notification mechanism has been tested through the experiment. We calculated the processing time of the system and it submitted to substantiate the reliability of the proposed service.

The rest of the paper is organized as follows. The following section overviews the related works of fire detection methods, and existing fire alarm systems. It also points out the deficiency of the existing systems. The Section 3 describes the proposed IoT mobile fire notification service architecture. Section 4 details the functional components in the proposed service and discusses the method for detection and notification in a video sequence. Section 5 illustrates the implementation environment and experiment results of the proposed service in this paper. Finally, section 6 is devoted to conclusion and pointing to some further issues.

\section{Related Works}

A number of fire detection systems which use different rules or methods to find fire objects in video sequence are developed by the researchers. In a general way, the flame or fire detection methods can be grouped into three categories: (1) color-based methods; (2) motion-based; (3) dynamic texture methods. Color detection is one of the earliest techniques, including rule-based methods and model-based methods for different color space, such as RGB, YCbCr, HSV, Lab and so on [3]. It's realized that RGB values of flame pixels are in the red-yellow color range. There are a lot of fire detection systems that used different rules to define fire objects in video sequence. To identify fire objects in a video sequence, different rules can be used in the fire detection systems. Color-based method is one of the most commonly used approaches in fire detection and there are many fire detection algorithms using color information. Motion information can also used to identify fire areas in the video. A moving object detection algorithm in the preprocessing phase was implemented in order to raise an alarm for possible fire in video [1]. Many researchers are committed to the research and development of fire detection system, here we give some examples to illustrate the development of states of the existing systems. In they proposed a mobile fire notification service based on network via the controller area network[4]. This paper describes the structure of a CAN-based system architecture and the design method of a CAN communication network to overcome the shortcoming of the conventional systems, such as weakness to noise. A wireless sensor network based fire detection system is proposed with the indoor localization capability [5]. They have designed and used open source hardware and software with ZigBee technology. For the indoor localization, 2D trilateration has been used to find a tagged object that is located on the specific surface. In fire detection system for libraries has been proposed that uses IRIS nodes and temperature sensors [6]. The designed system is implemented on the TinyOS platform and it is reported that the new system generates alarm react in about 3 seconds. However, these systems lack the capability to send a security alert to users when fire occurs. In they have proposed a multi-sensor fire detection and notification service using web-based technologies. GSM module has been applied to send fire security notification to the owners [7]. A fuzzy logic based approach is used to process sensing data from different fire detection sensors. It also provides web-based notification platform to allow remote monitoring of fire detection devices. A monitoring system of alarm for 
fire detection using Arduino that are integrated with buzzer, smoke sensor and a camera. A wireless monitoring system is also implemented to display all the data taken from the sensor and the camera [8]. In discusses a smart fire detection system using WSN and GSM communication to detect fire. Smoke and temperature sensors have been used to reduce the false positives that are a common fault of the conventional system [9]. SMS capability is also implemented to allow the occupants to interact with the fire detection system. Most of these systems utilize alarms to warn users when a fire occurs, however these systems have an obvious disadvantage that if the users are far away from the alarms, he may be unable to know the fire occurs. Some systems utilize GSM to send fire notification but GSM mobile is not always reliable due to network problems and the power requirement issues.

\section{Proposed Fire Detection and Notification Service Architecture}

Figure 1 illustrates the conceptual architecture of the proposed fire detection and warning system. The proposed service is composed of three main components. The main function of the web app server is to set connection with the IP camera and obtain the video streams from the camera. The web app server is able to capture the video stream and then perform the detection approach to track the fire flame in the video. After the fire flame is detected, the server start send notifications to the mobile app client. The app client is responsible for receiving the notification from the web server and it provides the functionality to display the video stream to users.

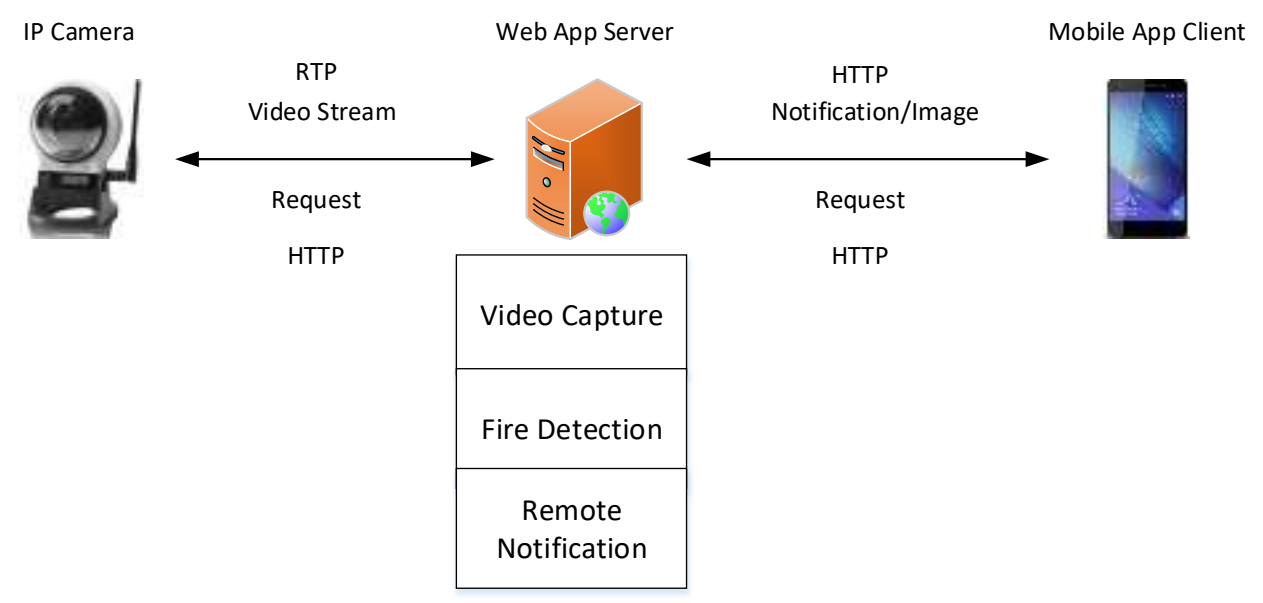

Figure 1. Architecture of the Intelligent Fire Detection and Notification Service

The block diagram for fire detection and tracking in the proposed service is illustrated in Figure 2. It consists of four components: acquisition of video sequences from the web camera, pre-processing of moving pixels in the current frame of a video, color feature extraction of moving pixels and tracking of the moving objects in the video sequence. The fundamental block diagram is shown below:

Information acquisition intends to acquire the video frames that are gained with the assistance of the camera. It is necessary to choose appropriate communication protocols to ensure the video streaming transmission under the minimum latency.

We have pre-processing procedure. The raw input from the IP camera is converted in a form that can be used and correspond to any other algorithms. In this paper, the color of the frame background of the video is into grey in pre-processing because the gray image requires less time in handling. 
Also, we have color feature extraction procedure. In object tracking, feature collection plays an import role. In the proposed approach, we track the target object utilizing the color feature, particularly red, green and blue shade. It is able to track the red, green and blue color objects in the video in the same way.

Here, bounding box is utilized to track in the video stream. As the object moves in the videos, the bounding box additionally moves with it and hence various features of area properties are obtained.

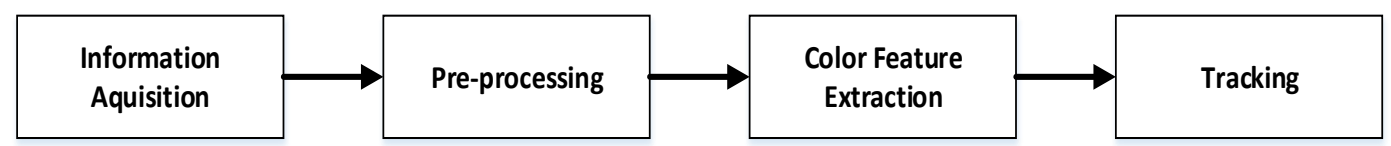

\section{Figure 2. Block Diagram for Fire Detection and Tracking in the Proposed Service}

\section{Functional Components of the Proposed Service}

Figure 3 describes the detailed structure of the web app server, the server consists of four functional components: UI module provides the input box for configuring the connection settings for the IP camera and the value settings for the RGB color space. Video display is a component used to play video streams comes from the IP camera and displays the processing results of the fire detection and tracking. Two sub modules is contained in the video control module. Video capture sets connection with the IP camera to get the video stream synchronized with the camera. Video saver provides the functionality to stop the connection with the IP camera and helps to save the video stream played. Fire detector module consists of flame detector and tracker, the flame detector applies the RGB based approach to track the defined color objects in the video and the flame tracker provides a bounding box that can automatically move to the detected area. The remote notification module is responsible for administering subscribers and sending notifications once the fire event occurs. The information of the subscriber is registered in the notification broker. The notification generator is utilized to create a notification message and the message is transmitted from the notification broker to the app client.

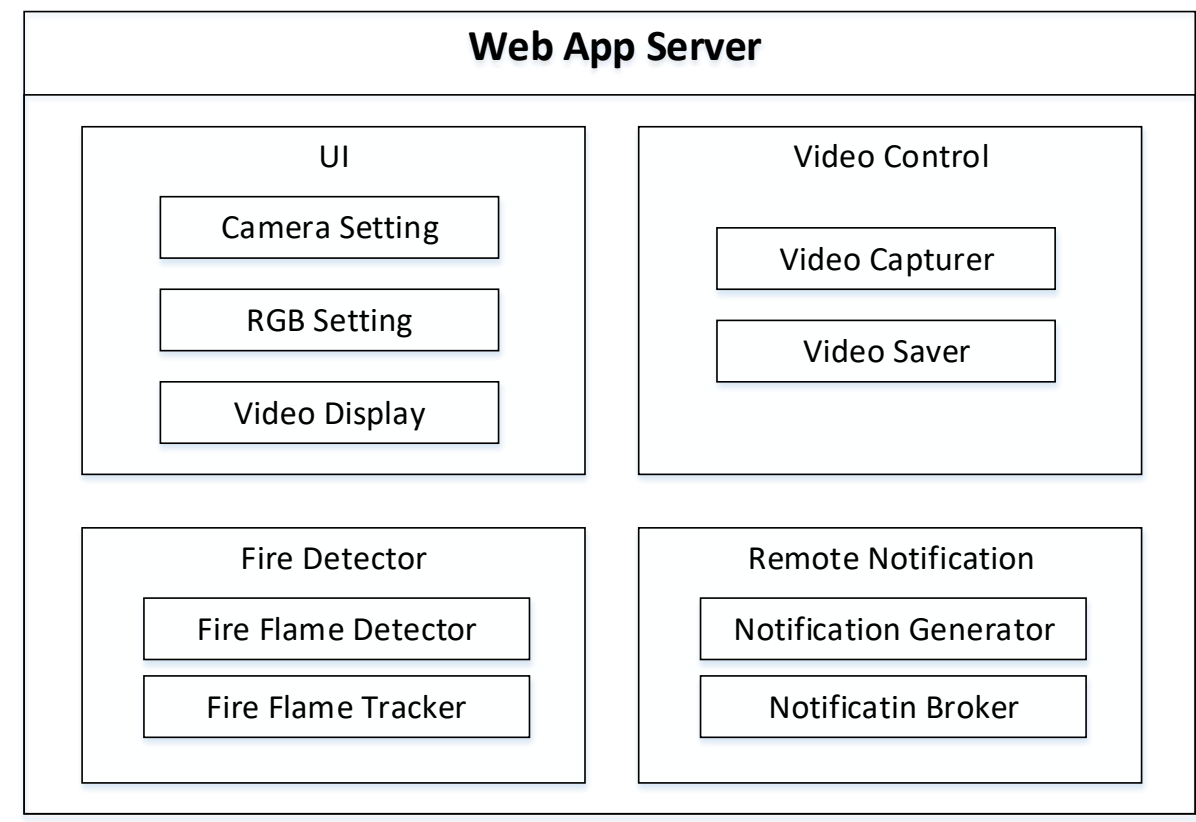

Figure 3. Functional Configuration of Web App Server 
Figure 4 represents the detailed structure of the mobile app client, the server consists of two functional components: UI module provides the view for playing the video stream and the control interface to interact with the web app server. The notification requester in the notification control module provides the ability to send a polling request to the web server while the notification receiver is used to receive the response information.

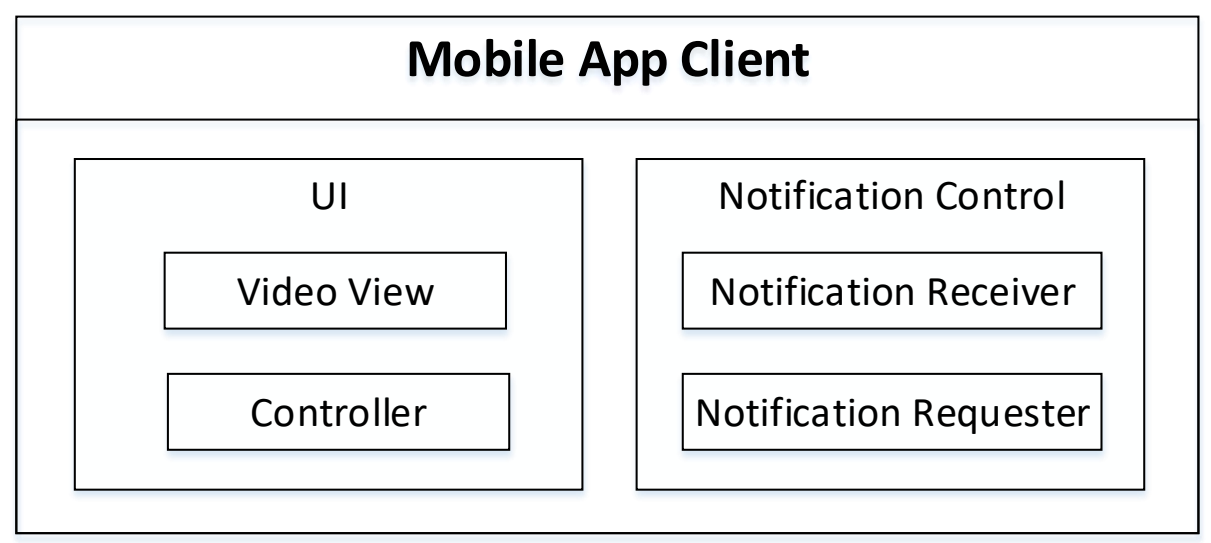

Figure 4. Functional Configuration of Mobile App Client

Figure 5 shows a procedure for fire detection and warning of the proposed service. When a user running the detection system, the basic configurations are needed for connecting the IP camera. If the connection with the IP camera is set successfully, the video stream will be delivered to the system and displayed to the user. Then the system begins to analyze the video stream to detect the flame objects. If the fire flame is detected, the system will stop capturing the video and save the captured video. Otherwise the system will continue capturing the video and perform detection method. After saving the captured video, the system will send out a notification message to the service subscriber. In this paper, the subscriber is a smart phone that has the capability to receive and display the video stream from the system.

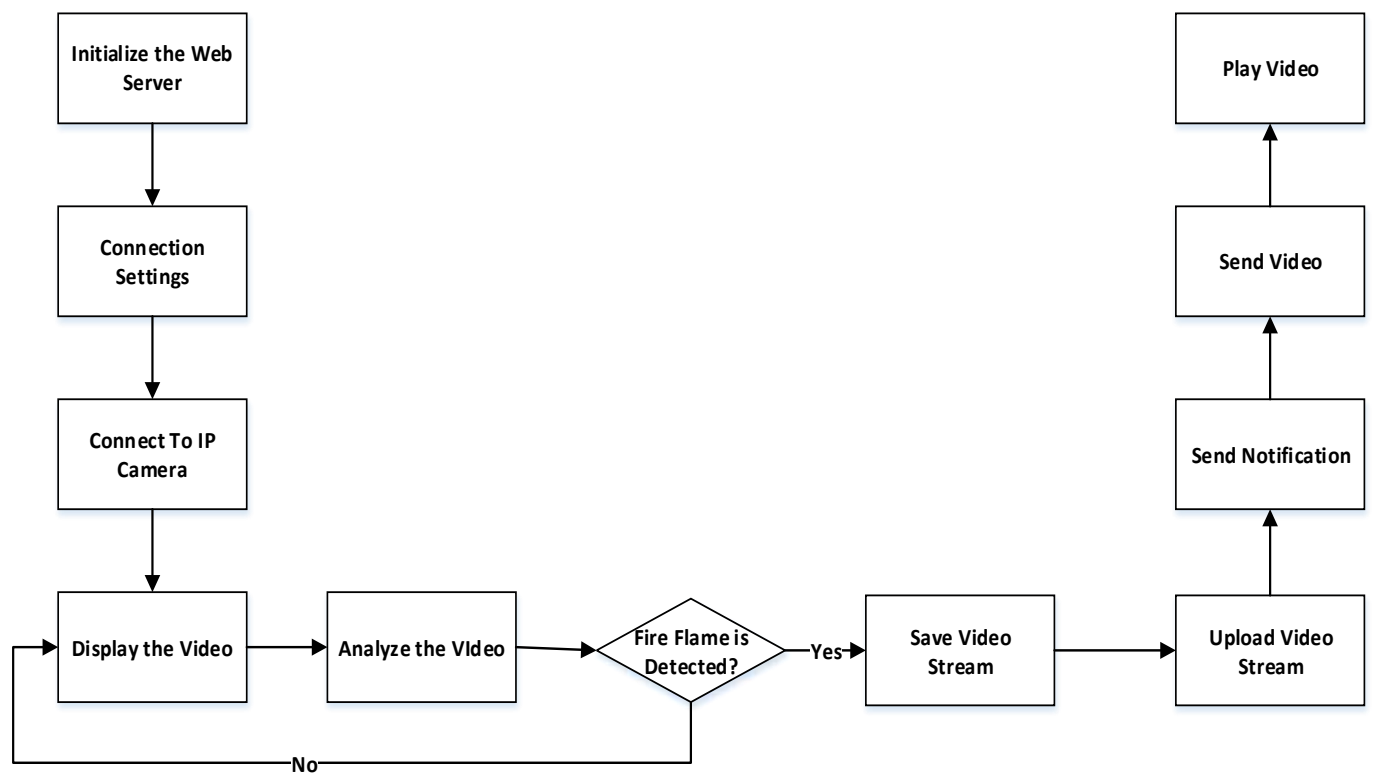

Figure 5. Fire Detection and Warning Procedure in the Proposed Service 
Figure 6 illustrates the operations between each components in the proposed service. After internalizing the app server, the user has an obligation to configure the connection settings of the IP camera. After the configuration is completed, the server starts to request the IP camera for connection. If the connection has succeeded, the video streams are transferred to the server and the user can check the video stream through the interface. The next step is to identify the values for each RGB color. Then the fire detection method is performed to detect the fire flame in the video. If the fire is detected, the server will stop playing the video and save the captured video stream. After that, the notification is performed to push warning messages to the notification subscriber. If the client receives the notification message, the user can request the video in the server then after the download is completed the client will play the video on the screen.

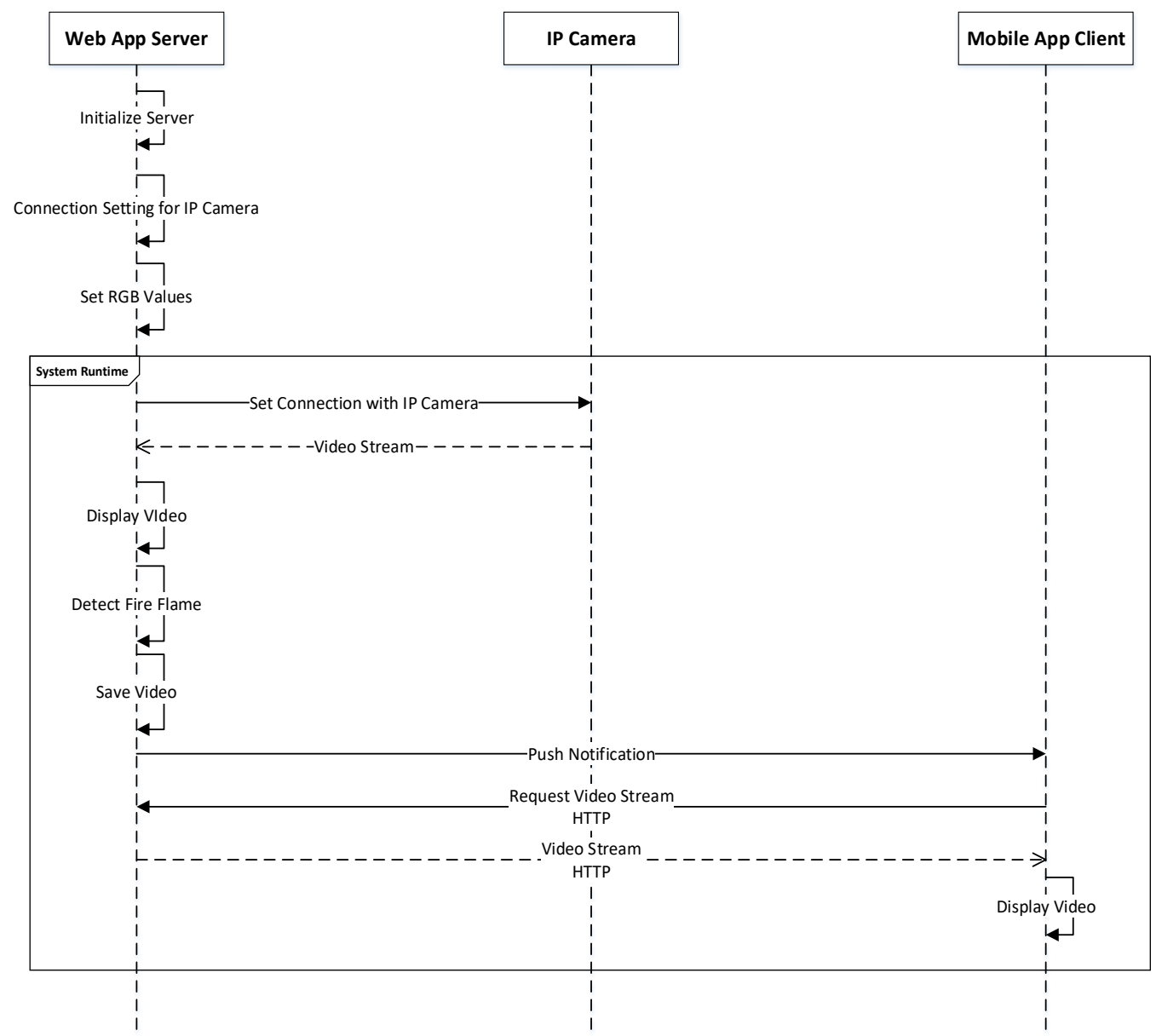

Figure 6. Sequence Diagram in the Proposed Service

\section{Implementation and Performance Evaluation}

The development environment of web app server is described in detail in Table 1. The server is developed with Visual Studio 2015 in Windows 7 Ultimate 64 bits. HTTP protocol has been used to support the data exchange between IP camera and the app client. We apply AForge.NET and Windows Forms libraries in the development. AForge.NET is an open source C\# framework designed for developers and researchers in the fields of image processing, neural networks, genetic algorithms, fuzzy logic, machine learning, robotics, etc [10]. 
Table 1. Development Environment of Web App Server

\begin{tabular}{|l|l|}
\hline \multicolumn{2}{|c|}{ Web App Server } \\
\hline Development Tool & Visual Studio Community 2015 \\
\hline Communication Protocol & HTTP \\
\hline Language & C\# \\
\hline Library & AForge.NET, Windows. Forms \\
\hline OS & Windows 7 Ultimate 64 bit \\
\hline
\end{tabular}

Table 2 represents the development environment of the mobile app client. This application is developed with Android Studio 2.2 in Windows 7 Ultimate 64 bits. In order to assist HTTP communication in the smart phone, we applied Google Volley library. The client application has been tested in the Vivo X5 Pro based on Android 5.0.

Table 2. Development Environment of Mobile App Client

\begin{tabular}{|l|l|}
\hline \multicolumn{2}{|c|}{ Mobile App Client } \\
\hline Development Tool & Android Studio 2.2 \\
\hline Communication Protocol & HTTP \\
\hline Language & Java, XML \\
\hline Library & Volley \\
\hline Hardware & Vivo X5 Pro (Android 5.0) \\
\hline
\end{tabular}

As shown in Figure 7, the web app server provides an UI for users to view the video stream from the IP camera in real time. On the right of the control interface, there is an input box for connecting settings of the IP camera. The IP address, id and the password are required set connection with the camera. Next to the camera setting box, there is a control bar for setting the range for each RGB color value. The user can drag the scroll bar for each color up and down to increase or decrease the value. On the left of the control interface, two video boxes are close to each, the left one is used to display the original video stream from the IP camera while the other one shows the processing results of the detected fire flame.

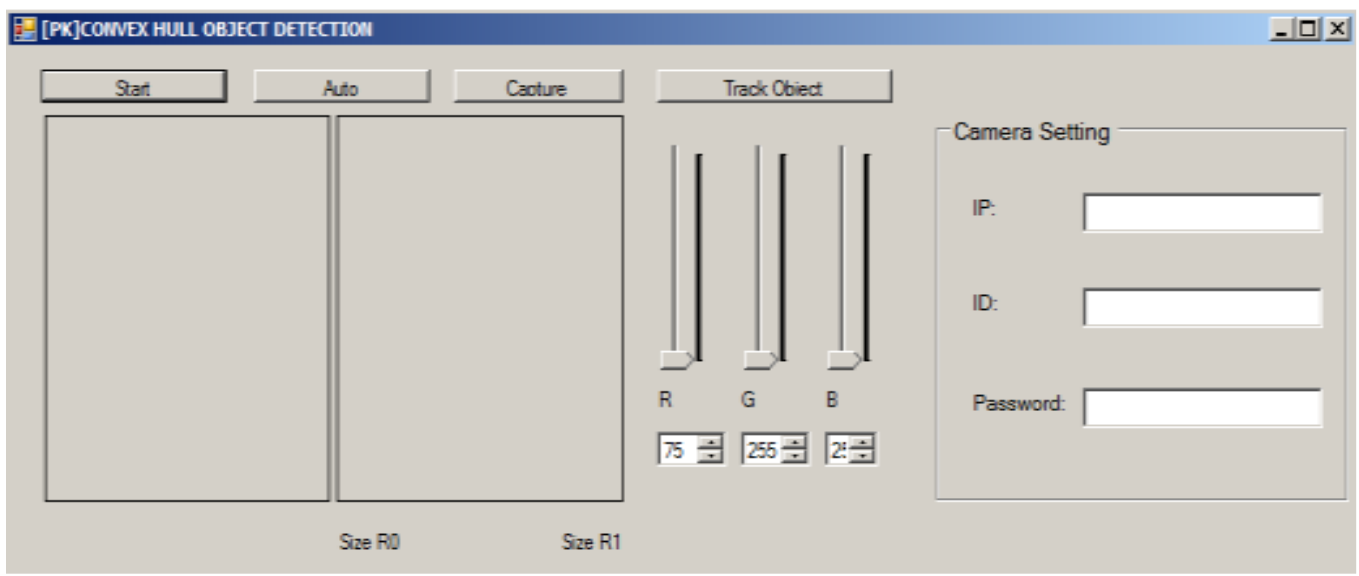

Figure 7. Control Interface of the Web App Server

Figure 8 represents the processing result of the fire detection and tracking in the web server. To connect with the IP camera, the IP address, id and the password info is typed in the input box. After that, the user presses the start to send a connection request to the IP camera. If the connection success, the video streams are returned to the web server and the video box on the left starts to play the video stream. Meanwhile, the video box on the 
right modifies the color of the video into grey. For getting the color features of the flame, we need to set the values for the red, green and blue color, in this paper, we set the red color to 140, green color to 240 and blue color to 0 . We have tried different settings and eventually found this setting is the most suitable. The flame and the lighter are detected within a bounding box in the videos box as shown in the figure. Till now, we have detected the fire flame in the video stream then we press the capture button on the interface and the server will save the video stream and starts to send notification to the client.

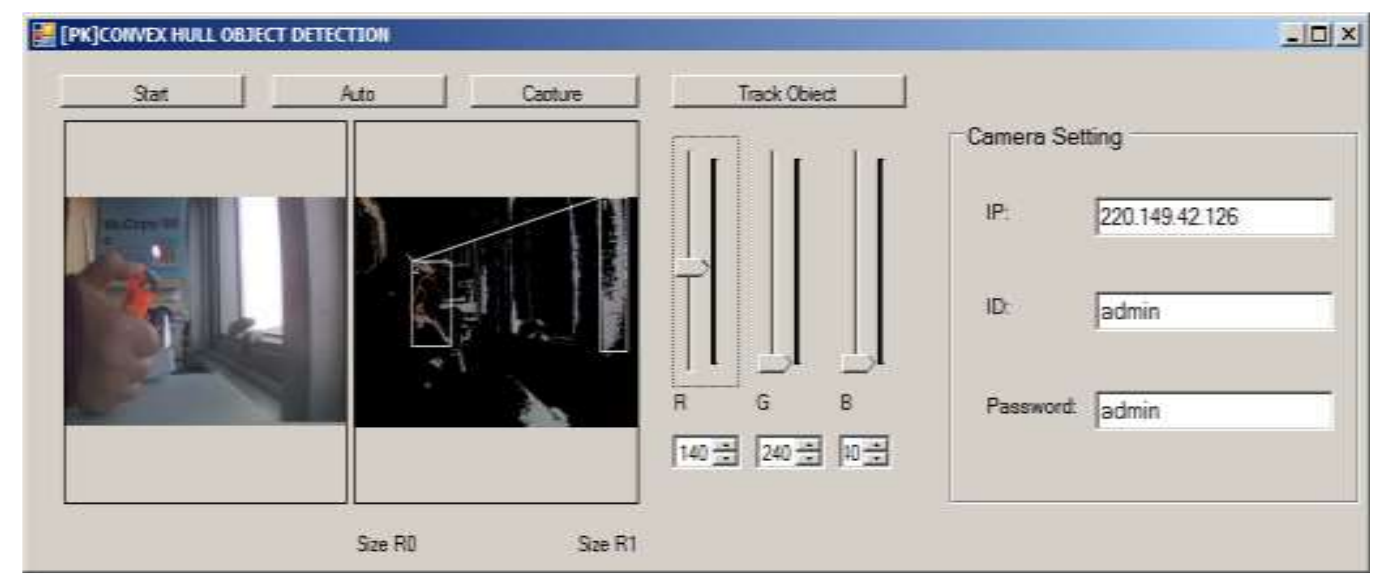

Figure 8. Process Result of the Detected Fire Flame

As shown in Figure 9, the mobile app client provides two buttons in the main interface. The app client sets up connection with the web server and start to wait for the response from the web server when user clicks the 'receive notification' button. The client initializes a polling request to the web server, the interval between each request is 5 seconds and the server returns a response immediately after receiving the request message and close the connection. If the client receives the response information, the 'view notification' button becomes active and it allows the client to receive the video sequence stored in the web server.

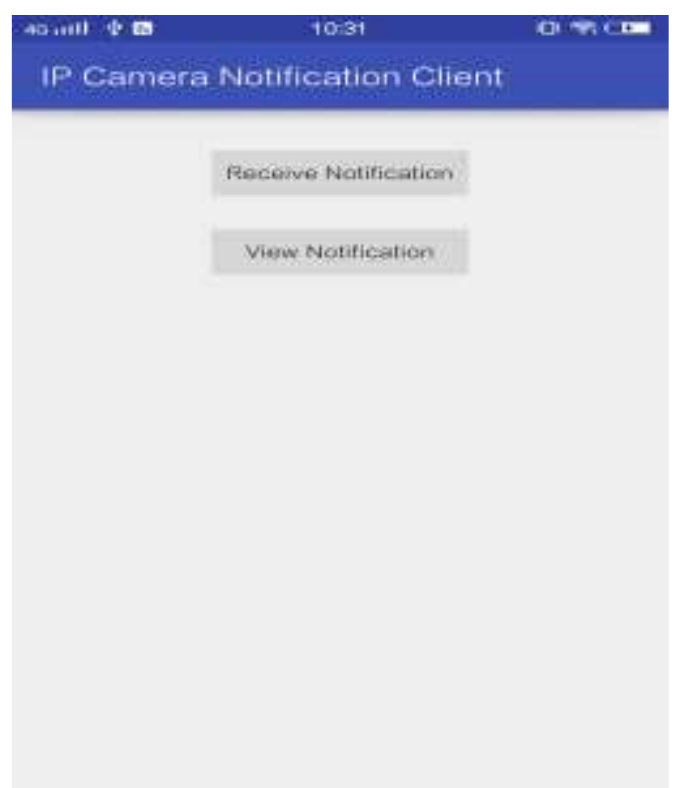

Figure 9. User Interface of the Mobile App Client 
Figure 10 represents the result of playing the video stream in mobile client. The app client requests the video stream from the web server and starts to download the contents. Generally, the costing time of this process depands on the network conditions and bandwidth. After the client completes the downloading, the video is automatically played in the smart phone.

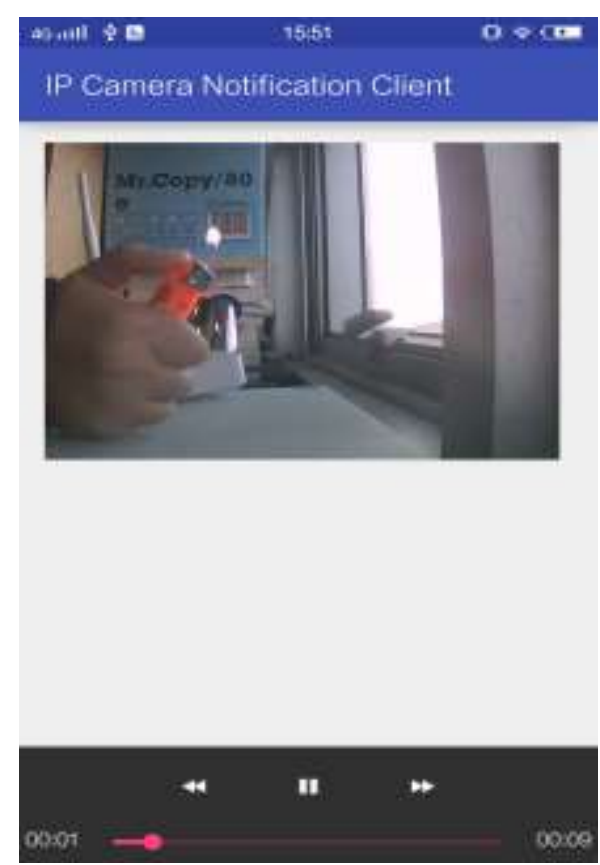

Figure 10. Monitoring the Video Stream in Mobile App Client

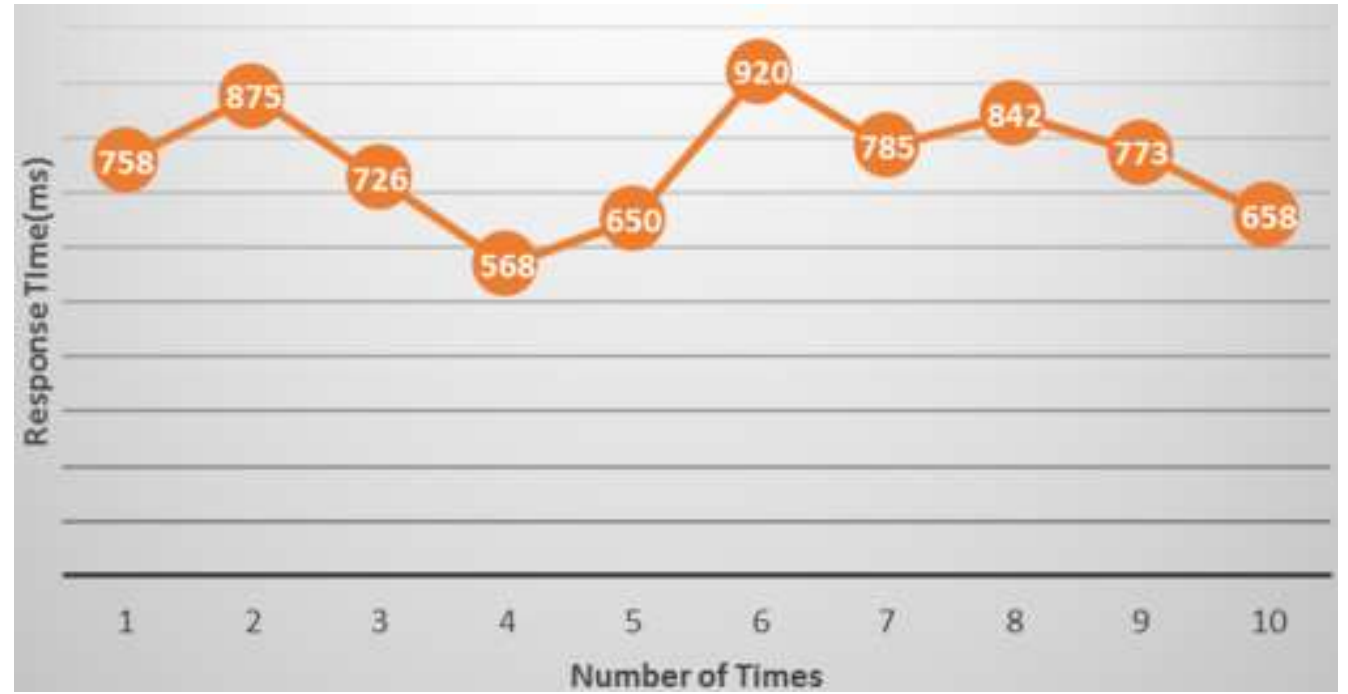

Figure 11. Processing Time Evaluation of the Proposed Service

This round trip time includes the latency time between the IP camera and the Web application server and the processing time for fire detection. But the cost time on downloading the video is not included because this time is based on the size of the video stream. Figure 11 illustrates the performance evaluation result of the proposed service. In order to obtain more accurate results, we have recorded the response time results ten times. According to the experiment, the average costs of the proposed service is 755.5 milliseconds. The processing time is such a low that it can be ignored and hardly been 
observed. The performance results verify that the proposed service has the ability of realtime fire detection and notification.

\section{Conclusion}

In this paper, we present a fire detection and notification service with an IP camera for smart home. The service can detect a fire from the physical fire behavior and warn people automatically before entering dangerous state. We use the detecting and tracking technique of fire flame using color feature extraction automatically. The service alerts users by pushing notification message; it also sends detection videos so that users can monitor the fire status in time remotely. With the increase of mobile users, there is a strong demand for managing great amount of mobile devices. New notification mechanism for managing group of users is needed to be investigated to satisfy compatibility of different mobile devices. According to the experimental results it is concluded that the current HTTP protocol is not very suitable for transmission of video content, we think that a different communication protocol of the study is very necessary.

\section{Acknowledgments}

This work was supported by Institute for Information \& communications Technology Promotion (IITP) grant funded by the Korea government (MSIT) (No.2017-0-00756, Development of interoperability and management technology of IoT system with heterogeneous ID mechanism), and this research was supported by the MSIT (Ministry of Science and ICT), Korea, under the ITRC (Information Technology Research Center) support program (IITP-2017-2014-0-00743) supervised by the IITP (Institute for Information \& communications Technology Promotion), Any correspondence related to this paper should be addressed to DoHyeun Kim.

\section{References}

[1] M. Hasan and M. Abdur Razzak, "An Automatic Fire Detection and Warning System under Home Video Surveillance", 2016 IEEE 12th International Colloquium on Signal Processing \& its Applications (CSPA2016), Melaka, Malaysia, (2016).

[2] K. Kavitha, A. Tejaswini and K. Kavitha, "VIBE: Background Detection and Subtraction for Image Sequences in Video", (IJCSIT) International Journal of Computer Science and Information Technologies, vol. 3, no. 5, (2012), pp. 5223-5226

[3] "Real-time object detection and Tracking using color feature and motion", Pritpal Singh, B.B.V.L. Deepak, Tanjot Sethi, Communications and Signal Processing (ICCSP), 2015 International Conference on the IEEE ICCSP.

[4] K. C. Lee and H. H. Lee, "Network-based fire-detection system via controller area network for smart home automation", IEEE Transactions on Consumer Electronics, vol. 50, issue 4, (2004), pp. 1093 1100 .

[5] R. Sowah, A. R. Ofoli, S. Krakani and S. Fiawoo, "A web-based communication module design of a real-time multi-sensor fire detection and Notification Service", 2014 IEEE Industry Application Society Annual Meeting.

[6] T. Islam, H. A. Rahman and M. Ahmed Syrus, "Fire detection system with indoor localization using ZigBee based wireless sensor network", 2015 International Conference on Informatics, Electronics \& Vision (ICIEV), (2015).

[7] S. Arslan, M. Challenger and O. Dagdeviren, "Wireless sensor network based fire detection system for libraries", Computer Science and Engineering (UBMK), 2017 International Conference on.

[8] M. Salihin Ahmad Azmil, N.Ya'Acob, K. Nizam Tahar and S. Seroja Sarnin, "Wireless fire detection monitoring system for fire and rescue application", Signal Processing \& Its Applications (CSPA) 2015 IEEE 11th International Colloquium on, IEEE, (2015), pp. 84-89.

[9] K.B. Deve, G.P. Hancke and B.J. Silva, "Design of a smart fire detection system", Industrial Electronics Society, IECON 2016 - 42nd Annual Conference of the IEEE.

[10] “AForge.NET", https://github.com/andrewkirillov/AForge.NET. 


\section{Authors}

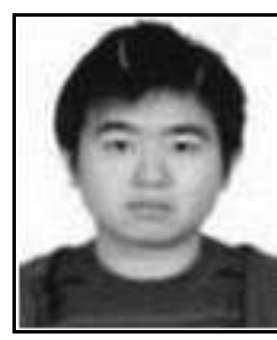

Lei Hang, he received the B.S. degree in computer engineering in 2015 and the M.S. degree from the Jejunu National University, Korea, in 2017. He is currently a Ph.D student at Jeju National University, South Korea. His area of interest is sensor networks, $\mathrm{M} 2 \mathrm{M} / \mathrm{IOT}$, intelligent service, and mobile computing.

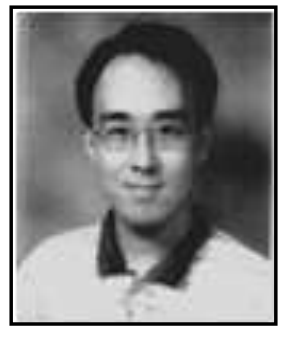

Do-Hyeun Kim, he received the B.S. degree in electronics engineering from the Kyungpook National University, Korea, in 1988, and the M.S. and Ph.D. degrees in information telecommunication the Kyungpook National University, Korea, in 1990 and 2000, respectively. He joined the Agency of Defense Development (ADD), from Match 1990 to April 1995. Since 2004, he has been with the Jeju National University, Korea, where he is currently a Professor of Department of Computer Engineering. From 2008 to 2009, he has been at the Queensland University of Technology, Australia, as a visiting researcher. His research interests include sensor networks, M2M/IOT, energy optimization and prediction, intelligent service, and mobile computing. 
International Journal of Control and Automation

Vol. 11, No. 1 (2018) 